\title{
On stability and reachability of perturbed positive systems
}

\author{
Begoña Cantó*, Carmen Coll and Elena Sánchez
}

\section{*Correspondence:}

bcanto@mat.upv.es

Instituto de Matemática

Multidisciplinar, Universitat

Politècnica de València, Camino de

Vera, 14, Valencia, 46022, Spain

\begin{abstract}
This paper deals mainly with the structural properties of positive reachability and stability. We focus our attention on positive discrete-time systems and analyze the behavior of these systems subject to some perturbation. The effects of permutation and similar transformations are discussed in order to determine the structure of the perturbation such that the closed-loop system is positively reachable and stable. Finally, the results are applied to Leslie's population model. The structure of the perturbation is shown such that the properties of the original system remain and an explicit expression of its set of positively reachable populations is given.
\end{abstract}

Keywords: positive linear system; M-matrix; nonnegative matrix; stability; reachability; perturbation

\section{Introduction}

Systems of difference equations with nonnegative coefficients are used as models in many fields in which the variables are subject to nonnegative restrictions. Examples of such applications can be found in [1-7]. One of the main aims in the study of real processes is to analyze if the system satisfies the stability property. However, often important properties such as stability and reachability are undetectable. Thus, it is important to know if the process disturbances can be attenuated by a feedback or if a trajectory will or will not reach a desired state using nonnegative controls. In [8] some results related to these topics are given.

Usually the system can be subject to disturbances. It is important to know what conditions must satisfy these disturbances in order to preserve the structure and properties that characterize our system. In this paper we consider a positive linear discrete-time system, stable and positively reachable. We propose the problem of determining what kind of perturbations can be used so that the closed-loop system maintains stability and positive reachability. Some results on the structure of the disturbances are given. Motivated by the application of the obtained results in some real processes, we focus on the case where the state matrix has a companion structure. In particular, we study the Leslie population model and we give the conditions for the system to be positively reachable, and we characterize the collection of perturbations under which the model remains stable.

The rest of the paper is organized as follows. Section 2 presents some results on stability of perturbed positive systems. In Section 3, we analyze the collection of disturbances in order for the closed-loop system to keep stability and also be positively reachable. Section 4 
gives a real application that illustrates the results provided in the paper. Finally, some final conclusions are given.

Before proceeding, we introduce some notation, definitions, and basic results. We recall, see [9], that a matrix $M$ is called nonnegative if all its entries are nonnegative and it is denoted by $M \geq O$. A matrix $M$ is an $M$-matrix if $M=s I-A$, where $A \geq O$ and $s \geq \rho(A)$, where $\rho(\cdot)$ denotes the spectral radius of a matrix, that is, the maximum modulus of its eigenvalues.

The stability of a matrix $M$ is equivalent to the condition $\rho(M)<1$. From the literature this property is also referred to as Schur stable matrix or convergent matrix. In [9] a characterization of this property for nonnegative matrices is given. Thus, a nonnegative matrix $M$ is stable if and only if $(I-M)^{-1} \geq O$. Finally, the norm $\|M\|_{1}$ is the maximum absolute column sum of $M$.

\section{Stability of perturbed positive systems}

Consider an invariant discrete-time system

$$
x(k+1)=A x(k), \quad k \geq 0,
$$

where the vector $x(k) \in \mathbb{R}^{n}$ and $A$ is a nonnegative matrix, that is, $A \geq O$.

Consider that the system is asymptotically stable, that is, $\rho(A)<1$, and let $\Delta \geq O$ be a perturbation matrix. We can prove that the perturbed system is asymptotically stable, $\rho(A+\Delta)<1$, if and only if $\rho\left(\Delta(I-A)^{-1}\right)<1$. This is established as follows.

Proposition 1 Let $A \geq O$ be with $\rho(A)<1$ and $\Delta \geq O$. The following assertions are equivalent:

(a) $\Delta(I-(A+\Delta))^{-1} \geq O$.

(b) $\rho\left(\Delta(I-A)^{-1}\right)<1$.

(c) $\rho(A+\Delta)<1$.

Proof Since $\rho(A)<1, H=\Delta(I-A)^{-1} \geq O$.

(a) $\Rightarrow$ (b) As $H \geq O, r=\rho(H)$ is an eigenvalue of $H$ with a nonnegative eigenvector $v$. From $H v=r v$ and noting that $\Delta(I-(A+\Delta))^{-1}=H(I-H)^{-1}$ we get $\Delta(I-(A+\Delta))^{-1} v=$ $\frac{r}{1-r} v \geq 0$. Thus $1-\rho(H)>0$.

(b) $\Rightarrow$ (c) If $\rho\left(\Delta(I-A)^{-1}\right)<1$, then $I-(A+\Delta)=\left(I-\Delta(I-A)^{-1}\right)(I-A)$ is an invertible $M$-matrix whose inverse matrix is nonnegative, and $I-A$ and $I-\Delta(I-A)^{-1}$ are $M$-matrices. Then $\rho(A+\Delta)<1$.

(c) $\Rightarrow$ (a) It is straightforward since $\rho(A+\Delta)<1$ and $\Delta \geq O$.

When the matrix $A$ has a companion structure, under similarity, we can take the entries of $A$ of the upper diagonal equal to 1 ,

$$
A=\left(\begin{array}{cccc}
a_{1} & 1 & \cdots & 0 \\
a_{2} & 0 & \cdots & 0 \\
\vdots & \vdots & \cdots & \vdots \\
a_{n-1} & 0 & \cdots & 1 \\
a_{n} & 0 & \cdots & 0
\end{array}\right) .
$$

This matrix satisfies $|I-A|=1-\sum_{j=1}^{n} a_{j}$. 
If the entries $\left\{a_{i} \geq 0, i=1, \ldots, n\right\}$ are perturbed, $a_{i}+\delta_{i}$ with $\delta_{i} \geq 0, i=1, \ldots, n$, the new perturbed matrix $A+\Delta$, with

$$
\Delta=\left(\begin{array}{cccc}
\delta_{1} & 0 & \cdots & 0 \\
\delta_{2} & 0 & \cdots & 0 \\
\vdots & \vdots & \cdots & \vdots \\
\delta_{n-1} & 0 & \cdots & 0 \\
\delta_{n} & 0 & \cdots & 0
\end{array}\right),
$$

satisfies the following result.

Proposition 2 Consider matrix $A$ as (1) and matrix $\Delta \geq O$ as (2), then $A+\Delta$ is asymptotically stable if and only if $\|\Delta\|_{1}<1-\|A\|_{1}$.

Proof From the structure of matrices $A$ and $\Delta$ we check

$$
\rho\left(\Delta(I-A)^{-1}\right)=\frac{\sum_{j=1}^{n} \delta_{j}}{|I-A|}
$$

Hence, $A+\Delta$ is asymptotically stable if and only if $\sum_{j=1}^{n} \delta_{j}<1-\sum_{j=1}^{n} a_{j}$, that is, $\|\Delta\|_{1}<$ $1-\|A\|_{1}$.

Note that, if $\delta_{i}=1, i=1, \ldots, n$, then $A+\Delta$ is not asymptotically stable. By definition of $A$ and $\Delta$, we can check that the matrix $\Delta(I-(A+\Delta))^{-1}$ is not a nonnegative matrix since all its entries are equal to $\frac{-1}{(n-1)+a_{1}+\cdots+a_{n}}<0$. From Proposition $1, \rho(A+\Delta) \geq 1$, then $A+\Delta$ is not asymptotically stable. On the other hand, the characterization of Proposition 2 suggests that the parameters of the perturbation must satisfy $0 \leq \delta_{i}<1, i=1, \ldots, n$.

\section{Stability and positive reachability of perturbed positive systems}

Now we fix our attention to a positive discrete-time control system,

$$
x(k+1)=A x(k)+B u(k), \quad k \geq 0,
$$

where the state vector $x(k) \in \mathbb{R}^{n}$, the control vector $u(k) \in \mathbb{R}^{m}$ with $m \leq n$, and $A$ and $B$ are nonnegative matrices, that is, $A, B \geq O$. This system is denoted by $(A, B) \geq O$ and it is a positive system since for all nonnegative initial states $x(0) \geq 0$ and for all nonnegative control or input sequences $\{u(j)\} \geq 0, j \geq 0$, the trajectory of the system is nonnegative.

Using a nonnegative feedback $u(k)=\Delta x(k), \Delta \geq O$, the closed-loop system is given by the state matrix $A+B \Delta$. If the initial system is asymptotically stable, $\rho(A)<1$, we want to obtain the conditions on $\Delta$ in order to ensure that the new closed-loop system is also asymptotically stable. In particular, if $\Delta=\delta S \geq O$ where $S=\left(I_{m} O\right)$ from Proposition 1 we can ensure that $A+B \Delta$ is asymptotically stable if and only if $\delta<\frac{1}{R}$ with $R=\rho\left(B S(I-A)^{-1}\right)$.

In several applications it will be important to reach a given state using an adequate control sequence. For example, some control functions are studied for linear discrete delay systems in $[10,11]$. By definition, $(A, B)$ is reachable if for every final state $x_{f} \in \mathbb{R}^{n}$ there exists a finite input sequence transferring the initial state to $x_{f}$. This property is 
known as reachability property and it is characterized by the range of reachability matrix $\mathrm{R}(A, B)=\left(B A B \cdots A^{n-1} B\right)$. Thus, $(A, B)$ is reachable if and only if the matrix $\mathrm{R}(A, B)$ has full rank. The set of all reachable states is the subspace generated by the independent linear columns of $\mathrm{R}(A, B)$. When the system is reachable this subspace is the space $\mathbb{R}^{n}$. But when the nonnegative restrictions are imposed new features arise and we have the concept of positive reachability property. The interest in this property is motivated by the large number of fields (bioengineering, economic modeling, biology, and behavioral science) in which it is always necessary that the inputs $u$ are also nonnegative. Thus, the system $(A, B) \geq O$ is positively reachable if for every final state $x_{f} \in \mathbb{R}_{+}^{n}$ there exists a finite nonnegative input sequence transferring the initial state to $x_{f}$.

This property was studied in $[12,13]$. Some results given in these works establish that this property holds if and only if the reachability matrix contains a monomial submatrix of order $n$. Recall that a monomial vector is a (nonzero) multiple of some canonical basis vector, and a monomial matrix $M$ is a matrix whose columns are distinct monomial vectors and can be decomposed as $M=D P$ where $D$ is a diagonal matrix and $P$ is a permutation matrix. In this case, the set of all positively reachable nonnegative states is the cone generated by the independent monomial columns of $\mathrm{R}(A, B)$. When the system is positively reachable this cone is $\mathbb{R}_{+}^{n}$.

It is widely known that two systems are similar if we can obtain one from the other by a change of base, $x(k)=T \hat{x}(k)$. Thus, system $(A, B)$ is similar to system $(\hat{A}, \hat{B})$ if there exists a nonsingular matrix $T$ such that $\hat{A}=T^{-1} A T$ and $\hat{B}=T^{-1} B$.

The general reachability property is preserved under similarity transformations, however, two similar positive systems are not necessarily both positively reachable. Then the concept of positive similar is introduced in the following way. Two positive systems $(A, B)$ and $(\hat{A}, \hat{B})$ are positively similar if there exists a square nonnegative monomial matrix $M$ satisfying $\hat{A}=M^{-1} A M$ and $\hat{B}=M^{-1} B$. In [14] it is established that the positive reachability property is transferred under positive similarity.

Moreover, in [12] the authors gave a positive reachability canonical form. This canonical form has an upper triangular block structure where the diagonal blocks are formed by cyclic, nilpotent, and companion submatrices. Using this canonical structure we consider the pair $(A, B)$

$$
A=\left(\begin{array}{cccc}
A_{1} & \Phi & \cdots & \Phi \\
O & A_{2} & \cdots & \Phi \\
\vdots & \vdots & \cdots & \vdots \\
O & O & \cdots & \Phi \\
O & O & \cdots & A_{h}
\end{array}\right), \quad B=\left(\begin{array}{c}
B_{1} \\
B_{2} \\
\vdots \\
B_{h-1} \\
B_{h}
\end{array}\right)
$$

with $A_{j} \in \mathbb{R}_{+}^{n_{j} \times n_{j}}$ companion matrix as (1) whose entries of the first column are $\left\{a_{i}^{j}, i=\right.$ $\left.1, \ldots, n_{j}\right\}, B_{j} \in \mathbb{R}_{+}^{n_{j} \times h}$ has all entries zero except the entry of position $\left(n_{j}, h-j+1\right)$ denoted by $b_{j}$, for all $j=1, \ldots, h$ and $\sum_{j=1}^{h} n_{j}=n$. Moreover, $\Phi \geq O$ only can have nonzero entries in the first column. This system is positively reachable since it satisfies the structure of the canonical form and it is easy to prove that the reachability matrix contains a monomial matrix of order $n$. 
From now on, without loss of generality we assume that the initial time is zero, because otherwise we just need to perform a change of variables first to transfer the initial state to zero.

Returning to the initial approach we want to study the invariance of both properties, stability and positive reachability, when the system is subjected to perturbations.

Proposition 3 Let $A, B \geq O$ be given as in (3). Consider the perturbation matrix $\Delta=$ $\left(\Delta_{1} \cdots \Delta_{h}\right)$ being $\Delta_{j}=\delta_{j} S_{j} \geq O$ and $S_{j} \in \mathbb{R}_{+}^{h \times n_{j}}$ has all entries zero except the entry of position $\left(h-j+1, n_{j}\right)$ which is equal to $1, j=1, \ldots, h$. Then:

(a) The perturbed system $(A+B \Delta, B)$ is also positively reachable from zero.

(b) If the system $(A, B)$ is asymptotically stable the perturbed system $(A+B \Delta, B)$ is asymptotically stable if and only if $\delta_{j}<\frac{1-\left\|A_{j}\right\|_{1}}{b_{j}}, j=1, \ldots, h$.

Proof

(a) To prove the positive reachability of the new system $(A+B \Delta)$, we construct its reachability matrix and it is easy to check that it has a monomial matrix of size $n \times n$.

(b) By the structure of $A, B$, and $\Delta$ we have

$$
\rho\left(B \Delta(I-A)^{-1}\right)=\max _{1 \leq j \leq n} \rho\left(B_{j} \Delta_{j}\left(I-A_{j}\right)^{-1}\right)=\max _{1 \leq j \leq n} \frac{b_{j} \delta_{j}}{\left|I-A_{j}\right|} .
$$

By Proposition 1 we find that the new system $(A+B \Delta)$ is asymptotically stable if and only if $\delta_{j}<\frac{1-\left\|A_{j}\right\|_{1}}{b_{j}}$, for all $j=1, \ldots, h$.

\section{Application to Leslie's population model}

Leslie's model is a discrete, age-structured model of population growth. It is used to model the changes in a population of organisms over a period of time. It is widely used in population ecology and demography to determine the growth of a population, as well as the age distribution within the population over time. There are a lot of studies on this model. To obtain more information on some applications from population matrix models in ecological and evolutionary studies see [15] and the references therein.

The Leslie model combines births and deaths in a single model and it is based on these hypotheses: (i) The age $x$ is a variable starting from 0 and subdivided into $n$ discrete age classes of size $h$. The age class $i$ corresponds to the ensemble of individuals whose ages satisfy $(i-1) h \leq x<i h, i=1, \ldots, n$. (ii) Time is a discrete variable denoted by $k$ and the time-step is equal to the duration of each age class. That is, from $k$ to $k+1$ all individuals go from class $i$ to $i+1$.

If we denote by $x(k)$ the number of individuals in each age class at time $k$ and by $u(k)$ the measure of immigration or stocking rate, then Leslie's model is given by

$$
x(k+1)=\bar{A} x(k)+\bar{B} u(k),
$$

where $\bar{A}$ represents the $n \times n$ Leslie matrix and $\bar{B}$ represents the number of individuals of age $i$ entering the system per unit of control,

$$
\bar{A}=\left(\begin{array}{cccc}
f_{1} & f_{2} & \cdots & f_{n} \\
s_{1} & & & 0 \\
& \ddots & & \vdots \\
& & s_{n-1} & 0
\end{array}\right), \quad \bar{B}=\left(\begin{array}{c}
b \\
0 \\
\vdots \\
0
\end{array}\right),
$$


where the entries of the first row of the matrix $\bar{A}$ are given by the fertility, $f_{i}$, and the subdiagonal is given by the survival, $s_{i}$; and there are zeros elsewhere [16]. The fertility and survival rates are generally referred to as vital rates. The entry $b$ represents the fertility from an extern input [17].

The eigenstructure of the matrix $\bar{A}$ gives much information on the model. Thus, the dominant eigenvalue $\lambda$ determines the population growth in the long run. The other eigenvalues determine the transient dynamics of the population. When $\lambda=1$ the population is stationary, when $\lambda>1$ there is an over-population, and when $\lambda<1$ the population decreases. On the other hand, the right eigenvectors include the stable age distribution and the left eigenvectors include the reproductive value [18].

In addition, the pair $(\bar{A}, \bar{B})$ is similar to the pair $(A, B)$ via the diagonal matrix $S=$ $\operatorname{diag}\left(1, s_{1}, s_{1} s_{2}, \ldots, s_{1} \cdots s_{n-1}\right)$ where $A=S^{-1} \bar{A} S$ and $B=S^{-1} \bar{B}$. This process is represented by the following discrete-time system:

$$
x(k+1)=A x(k)+B u(k)
$$

with

$$
A=\left(\begin{array}{cccc}
a_{1} & \cdots & a_{n-1} & a_{n} \\
1 & \cdots & 0 & 0 \\
\vdots & \cdots & \vdots & \vdots \\
0 & \cdots & 0 & 0 \\
0 & \cdots & 1 & 0
\end{array}\right), \quad B=\left(\begin{array}{c}
b \\
0 \\
\vdots \\
0
\end{array}\right)
$$

where $a_{1}=f_{1}, a_{j}=f_{j} \prod_{i=1}^{j-1} s_{i}, j=2, \ldots, n$.

Since $A$ and $B$ are nonnegative matrices we have a positive system. In addition, the system is reachable since the reachability matrix $\mathrm{R}(A, B)$ has full rank but the problem is that we cannot ensure that the used control is nonnegative. That is, we cannot assert that we can achieve a certain population from nonnegative controls. Maybe some nonnegative states can be reached by means of nonnegative inputs but not all because the system is not positively reachable, since $\mathrm{R}(A, B)$ does not contain a monomial submatrix of order $n$. To analyze this problem we will use the results of the above sections. First, we give some comments about the transformations that allow us to obtain the system of interest.

We define $T_{z}=(I-N)$ and $T=P T_{z}$ where $P$ is the antidiagonal permutation matrix,

$$
N=\left(\begin{array}{ccccc}
0 & a_{1} & \cdots & a_{n-2} & a_{n-1} \\
0 & 0 & \cdots & a_{n-3} & a_{n-2} \\
\vdots & \vdots & \cdots & \vdots & \vdots \\
0 & 0 & \cdots & 0 & a_{1} \\
0 & 0 & \cdots & 0 & 0
\end{array}\right) .
$$

Since $N$ is a nilpotent matrix, $\rho(N)<1$ and $T_{z}$ is an invertible $M$-matrix with $T_{z}^{-1}=$ $\sum_{i=0}^{n-1} N^{i}$. Thus, $T_{z}^{-1}$ and $T^{-1}$ are nonnegative matrices.

The set of positively reachable states is given in the following result. 
Proposition 4 The set of the population states which can be obtained in Leslie's population model from a nonnegative control sequence is the cone

$$
\mathcal{X}=\left\langle T_{z}^{-1} e_{1}, \ldots, T_{z}^{-1} e_{n}\right\rangle
$$

where $e_{i}$ is the ith canonical vector and $T_{z}=I-N$ with $N$ as (6).

Proof First, we observe that the system $(A, B)$ given in (5) is similar to the system $(\hat{A}, \hat{B})$ where

$$
\hat{A}=\left(\begin{array}{cccc}
a_{1} & 1 & \cdots & 0 \\
a_{2} & 0 & \cdots & 0 \\
\vdots & \vdots & \cdots & \vdots \\
a_{n-1} & 0 & \cdots & 1 \\
a_{n} & 0 & \cdots & 0
\end{array}\right), \quad \hat{B}=\left(\begin{array}{c}
0 \\
0 \\
\vdots \\
b
\end{array}\right)
$$

by means of the transformation matrix $T$ with $T=P T_{z}$ where $P$ is the antidiagonal permutation matrix.

As the reachability matrix of this system $(\hat{A}, \hat{B})$ contains a monomial submatrix of order $n$, the system is positively reachable. Hence, we can ensure that for all nonnegative state $\hat{x}$ there exists a nonnegative sequence of control $\mathbf{u}=(u(n-1) \cdots u(1) u(0))^{T} \geq O$ such that

$$
\mathrm{R}(\hat{B}, \hat{A}) \mathbf{u}=\hat{x}
$$

Then $\mathrm{R}(B, A) \mathbf{u}=T^{-1} \hat{x}$. So, $x$ is reachable by means of a nonnegative control sequence if and only if $T x$ is nonnegative.

Summarizing the previous comments, in the system $(A, B)$ a state $x$ is reachable by means of a nonnegative control sequence if and only if there exists $\hat{x} \geq 0$ such that $x=T^{-1} \hat{x}$. Thus, the set of positively reachable states is the image of the application $T^{-1}$ restricted to $\mathcal{R}_{+}^{n}$. From $T_{z}=I-N$ and $T_{z} \geq O$ we see that $\left\{T^{-1} e_{j}, j=1, \ldots, n\right\}$ are independent linear nonnegative vectors and they generate the cone of the reachable states by a nonnegative control sequence

$$
\begin{aligned}
\mathcal{X} & =\left\{x \in \mathcal{R}^{n} / \exists \hat{x} \in \mathcal{R}_{+}^{n}, x=T^{-1} \hat{x}\right\} \\
& =\left\langle T^{-1} e_{1}, \ldots, T^{-1} e_{n}\right\rangle=\left\langle T_{z}^{-1} e_{1}, \ldots, T_{z}^{-1} e_{n}\right\rangle .
\end{aligned}
$$

Note that using the expression of matrix $T_{z}^{-1}=\sum_{i=0}^{n-1} N^{i}$ we can write

$$
\mathcal{X}=\left\langle e_{1},(I+N) e_{2}, \ldots, \sum_{j=0}^{i-1} N^{j} e_{i}, \ldots, \sum_{j=0}^{n-1} N^{j} e_{n}\right\rangle \text {. }
$$

Moreover, a specific population $x=\left(x_{1} x_{2} \cdots x_{n}\right)^{T}$ can be obtained in Leslie's population model from a nonnegative control sequence if and only if $x_{n} \geq 0$ and

$$
x_{n-i} \geq \sum_{k=1}^{i} a_{k} x_{n-i+k}, \quad i=1, \ldots, n-1 .
$$


Now, we study Leslie's model submitted to some kind of perturbations and we analyze the reachability and stability properties. At this point we should discuss how the structure of the disturbance is such that the properties of the initial system remain, that is, to be stable and to have the same set of positively reachable states.

If we want to keep the same set of the reachable states using a nonnegative control sequence, then we only can consider perturbations of the kind $\Delta=\left(\begin{array}{lll}0 & 0 & \cdots\end{array}\right)$, with $\delta \geq 0$. Thereby we see that a similar perturbed system, $\hat{A}+\hat{B} \hat{\Delta}$, with $\hat{A}=T A T^{-1}, \hat{B}=T B$ and $\hat{\Delta}=\Delta T^{-1}$, has a structure as (8). Then it is sufficient to apply Proposition 3 for $h=1$ to prove that the positive reachability property is preserved. In the same way as Proposition 4 we can establish that the set of positively reachable population is given by (7).

To study the stability of the closed-loop perturbed system it is sufficient to analyze the spectral radius of the matrix $\hat{A}+\hat{B} \hat{\Delta}$. Applying the item (b) of Proposition 3 to this matrix we obtain $\rho(A+B \Delta)=\rho(\hat{A}+\hat{B} \hat{\Delta})<1$ if and only if $\delta<\frac{|I-A|}{b}$.

These results are summarized in the following proposition.

Proposition 5 Let $A, B \geq O$ be given as in (5). Consider the perturbation matrix $\Delta=$ $(00 \cdots \delta)$ such that $\delta \geq 0$. Then:

(a) The perturbed system $(A+B \Delta, B)$ has the same cone of positively reachable states from zero than $(A, B)$.

(b) If the system $(A, B)$ is asymptotically stable then the perturbed system $(A+B \Delta, B)$ is asymptotically stable if and only if $\delta<\frac{|I-A|}{b}$.

The obtained results in this section can be extended to a population with several groups or types of individuals where the group $G_{i}$ can also receive births from the rest of the groups $G_{j}, j>i$. Without loss of generality, we can study the results for the case of a species with two types or groups of individuals $G_{1}$ and $G_{2}$ so that one of them, the $G_{2}$ group, also provides $G_{1}$ group births. Then we see that the process is modeled by a system $(\bar{A}, \bar{B})$,

$$
\bar{A}=\left(\begin{array}{cc}
\bar{A}_{1} & \bar{\Phi} \\
O & \bar{A}_{2}
\end{array}\right), \quad \bar{B}=\left(\begin{array}{l}
\bar{B}_{1} \\
\bar{B}_{2}
\end{array}\right),
$$

where the matrix blocks are defined as in (4) and $\bar{\Phi}$ represents the connection between the two groups. After applying the appropriate transformation $S=\operatorname{diag}\left(S_{1}, S_{2}\right)$ where $S_{j}$, $j=1,2$, is constructed as the transformation matrix used for system (4), the system $(\bar{A}, \bar{B})$ is transformed into the system $(A, B)$

$$
A=\left(\begin{array}{cc}
A_{1} & \Phi \\
O & A_{2}
\end{array}\right), \quad B=\left(\begin{array}{l}
B_{1} \\
B_{2}
\end{array}\right),
$$

where for each $j=1,2, A_{j} \in \mathbb{R}_{+}^{n_{j} \times n_{j}}$ is a companion matrix as (5) whose entries of the first row $\operatorname{are}\left\{a_{i}^{j}, i=1, \ldots, n_{j}\right\}, a_{1}^{j}=f_{1}^{j}, a_{l}^{j}=f_{l}^{j} \prod_{i=1}^{l-1} s_{i}^{j}, l=2, \ldots, n_{j}$, where $\left\{f_{i}^{j}, i=1, \ldots, n_{j}\right\}$ and $\left\{s_{i}^{j}, i=\right.$ $\left.1, \ldots, n_{j-1}\right\}$ are the fertility and survival coefficients of the type or group $G_{j}$, respectively. Moreover, $B_{j} \in \mathbb{R}_{+}^{n_{j} \times 2}$ has all entries zero except the $\left(n_{j-1}+1,3-j\right)$-entry denoted by $b_{j}$ and $n_{1}+n_{2}=n$. Moreover, in this case we consider the matrix $\Phi \geq O$ has only one nonzero element in position $\left(1, n_{1}\right)$, given by $\varphi \prod_{i=1}^{n_{2}-1} s_{i}^{j}$ with $\varphi$ the fertility coefficient from the last age class of the group $G_{2}$ going to group $G_{1}$. 
Using the transformation matrix $T=\operatorname{diag}\left(T_{1}, T_{2}\right)$ where $T_{j}=P\left(I-N_{j}\right), j=1,2$, we obtain a system $(\hat{A}, \hat{B})$ as $(3)$. Then, applying the results on positive reachability and stability when the system is submitted to a perturbation, we find that the set $\mathcal{X}$ of the population states of the Leslie population model (11) which can be reached from zero using a nonnegative control sequence is the cone

$$
\begin{aligned}
& \mathcal{X}=\mathcal{X}_{1} \oplus \mathcal{X}_{2}, \\
& \mathcal{X}_{1}=\left\{\left(\begin{array}{ll}
x_{1}^{T} & 0
\end{array}\right)^{T} / x_{1} \in \tilde{\mathcal{X}}_{1}\right\} \text { and } \mathcal{X}_{2}=\left\{\left(\begin{array}{ll}
0 & x_{2}^{T}
\end{array}\right)^{T} / x_{2} \in \tilde{\mathcal{X}}_{2}\right\}
\end{aligned}
$$

where $\tilde{\mathcal{X}}_{j}$, constructed as in (9), are $\tilde{\mathcal{X}}_{j}=\left\langle e_{1},\left(I+N_{j}\right) e_{2}, \ldots, \sum_{i=0}^{n_{j}-1} N_{1}^{i} e_{n_{j}}\right\rangle$, where $T_{j}=P\left(I-N_{j}\right)$, $j=1,2$.

If we consider the perturbation matrix $\Delta=\left(\Delta_{1} \Delta_{2}\right)$ being $\Delta_{j}=\delta_{j} S_{j} \geq O$ such that $S_{j} \in$ $\mathbb{R}_{+}^{2 \times n_{j}}$ has only one nonzero element in position $\left(3-j, n_{j}\right)$ equal to $1, j=1,2$, then:

(a) The perturbed system $(A+B \Delta, B)$ has the same cone of positively reachable states from zero as $(A, B)$.

(b) If the system $(A, B)$ is asymptotically stable then the perturbed system $(A+B \Delta, B)$ is asymptotically stable if and only if $\delta_{j}<\frac{\left|I-A_{j}\right|}{b_{j}}, j=1,2$.

To clarify we give the following example.

Example 1 Consider a population with two groups or types of individuals where the type or group $G_{1}$ can also receive births from the group $G_{2}$, where each of them has four age classes with the following fertility $f_{l}^{j}$, survival $s_{l}^{j}$, and fertility from an extern input $b_{j}$, with coefficients

$$
\begin{aligned}
& f_{1}^{1}=0, \quad f_{2}^{1}=1, \quad f_{3}^{1}=4, \\
& s_{1}^{1}=0.3, \quad s_{2}^{1}=0.2, \quad s_{3}^{1}=0.4, \quad b_{1}=1, \\
& f_{1}^{2}=0, \quad f_{2}^{2}=1, \quad f_{3}^{2}=3, \\
& s_{1}^{2}=0.4, \quad s_{2}^{2}=0.2, \quad s_{3}^{2}=0.3, \quad b_{2}=1,
\end{aligned}
$$

and the fertility coefficient from the last age class of the group $G_{2}$ to group $G_{1}$ is $\varphi=3$.

By the above results, a state is positively reachable if $x-A^{n-1} x_{0}$ is in the cone (12). For instance, if we consider an initial population

$$
x_{0}=\left(\begin{array}{llllll}
330 & 290 & 210 & 1,030 & 300 & 200
\end{array}\right)^{T}
$$

in Leslie's model (11), a population state is positively reachable if there exists a nonnegative control such that $x=A^{n-1} x_{0}+\mathrm{R}(A, B) \mathbf{u}$, or equivalently, if $x-A^{n-1} x_{0}$ is in the cone

$$
\begin{aligned}
& \mathcal{X}=\left\{\left(\begin{array}{ll}
x_{1}^{T} & 0
\end{array}\right)^{T} / x_{1} \in \tilde{\mathcal{X}}_{1}\right\} \oplus\left\{\left(\begin{array}{ll}
0 & x_{2}^{T}
\end{array}\right)^{T} / x_{2} \in \tilde{\mathcal{X}}_{2}\right\}, \\
& \tilde{\mathcal{X}}_{1}=\left\langle e_{1}, e_{2}, 0.3 e_{1}+e_{3}\right\rangle \\
& \tilde{\mathcal{X}}_{2}=\left\langle e_{1}, e_{2}, 0.4 e_{1}+e_{3}\right\rangle
\end{aligned}
$$

Note that to obtain the cone $\mathcal{X}$ we use the conditions given in (10). Graphically the cones $\tilde{\mathcal{X}}_{1}$ and $\tilde{\mathcal{X}}_{2}$ would be as in Figure 1 . 


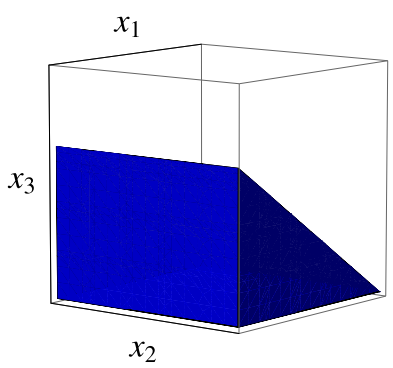

(a)

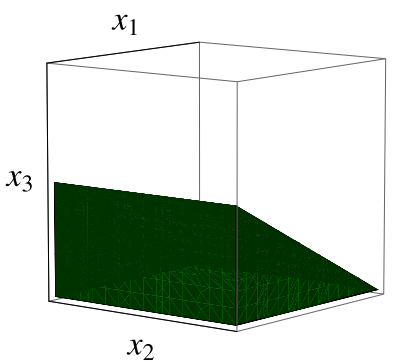

(b)

Figure 1 Positively reachable population from 0 in Leslie's model (11). (a) Positive reachability cone of $G_{1}:\left\{x \in \mathbb{R}^{3}: x_{1} \geq 0.3 x_{3}, x_{3}, x_{2} \geq 0\right\}$. (b) Positive reachability cone of $G_{2}:\left\{x \in \mathbb{R}^{3}: x_{1} \geq 0.4 x_{3}, x_{3}, x_{2} \geq 0\right\}$.

Using the transformation matrix $T$ we obtain the system $(\hat{A}, \hat{B})$ given by (11),

$$
\begin{array}{ll}
\hat{A}_{1}=\left(\begin{array}{ccc}
0 & 1 & 0 \\
0.3 & 0 & 1 \\
0.24 & 0 & 0
\end{array}\right), \quad \hat{A}_{2}=\left(\begin{array}{ccc}
0 & 1 & 0 \\
0.4 & 0 & 1 \\
0.24 & 0 & 0
\end{array}\right), \\
\hat{B}_{1}=\left(\begin{array}{ll}
0 & 0 \\
0 & 0 \\
0 & 1
\end{array}\right), & \hat{B}_{2}=\left(\begin{array}{ll}
0 & 0 \\
0 & 0 \\
1 & 0
\end{array}\right) .
\end{array}
$$

In fact this system is positively reachable and the nonnegative sequence control to reach any state of $\mathcal{X}$ can be calculated using this system. If, for example, we want to reach the population

$$
x=\left(\begin{array}{llllll}
490 & 380 & 380 & 1,300 & 600 & 1,100
\end{array}\right)^{T},
$$

which satisfies $\hat{x}-\hat{A}^{2} \hat{x}_{0} \geq 0$, it is sufficient to increase births by means of an input calculated as

$$
\mathbf{u}=\mathrm{R}^{-1}(\hat{B}, \hat{A})\left(\hat{x}-\hat{A}^{2} \hat{x}_{0}\right)
$$

where $\mathbf{u}=\left(u^{T}(2) u^{T}(1) u^{T}(0)\right)^{T}$ with $u(j)=\left(u_{1}^{T}(j) u_{2}^{T}(j)\right), j=0,1,2$. Then the desired population is obtained using the control or input of births at step $j$ and each group $G_{j}$; see Table 1.

Now, we observe that the system $(A, B)$ is asymptotically stable since $\rho(A)=0.83<1$. If we consider a perturbation

$$
\Delta=\left(\begin{array}{cccccc}
0 & 0 & 0 & 0 & 0 & \delta_{2} \\
0 & 0 & \delta_{1} & 0 & 0 & 0
\end{array}\right),
$$

Table 1 Inputs of births at step $j=0,1,2$ in each group $G_{1}$ and $G_{2}$

\begin{tabular}{rrr}
\hline $\boldsymbol{j}$ & $\boldsymbol{G}_{\mathbf{1}}$ & \multicolumn{1}{c}{$\boldsymbol{G}_{\mathbf{2}}$} \\
\hline 0 & 70 & 50 \\
1 & 432 & 195 \\
2 & 788 & 234 \\
\hline
\end{tabular}


the system $(A+B \Delta, B)$ has the same cone of positively reachable states as $(A, B)$ and taking $\delta_{1}<\frac{\left|I-A_{1}\right|}{b_{1}}=7$ and $\delta_{2}<\frac{\left|I-A_{2}\right|}{b_{2}}=4$ we can ensure that the perturbed system $(A+B \Delta, B)$ is also asymptotically stable. For instance if $\delta_{1}=6$ and $\delta_{2}=3$ we have $\rho(A+B \Delta)=0.96<1$.

\section{Conclusions}

Discrete-time positive systems are quite frequent in science and engineering. We consider the problem of determining the structure of a perturbation such that a perturbed positive discrete-time system has the positive reachability and stability properties. In the general model, to solve this problem the structure of the positive reachability canonical form introduced in [12] and a positive similarity transformation are used. Leslie's population model is analyzed. It is a discrete-time positive system, it is reachable, since the reachability matrix $\mathrm{R}(A, B)$ has full rank, but it is not positively reachable, since $\mathrm{R}(A, B)$ does not contain a monomial submatrix of order $n$. The explicit expression of the cone of the population states is shown which can be obtained in Leslie's population model from a nonnegative control sequence. Finally, a numerical example is given to clarify the obtained results.

\section{Competing interests}

The authors declare that they have no competing interests.

\section{Authors' contributions}

All authors completed the paper together. All authors read and approved the final manuscript.

\section{Acknowledgements}

The authors would like to thank the referee and the associate editor for their very helpful suggestions. This work has been partially supported by Spanish Grant MTM2013-43678-P.

Received: 17 July 2014 Accepted: 11 November 2014 Published: 26 Nov 2014

\section{References}

1. Cantó, B, Coll, C, Sánchez, E: Parameter identification of a class of economical models. Discrete Dyn. Nat. Soc. 2010, Article ID 408346 (2010)

2. Cao, H, Zhou, Y: The discrete age-structured SEIT model with application to tuberculosis transmission in China. Math. Comput. Model. 55, 385-395 (2012)

3. Coll, C, Herrero, A, Sánchez, E, Thome, N: A dynamic model for a study of diabetes. Math. Comput. Model. 50, 713-716 (2009)

4. Emmert, HE, Allen, LSJ: Population persistence and extinction in a discrete-time, stage-structured epidemic model. J. Differ. Equ. Appl. 10, 1177-1199 (2004)

5. Li, CK Schneider, H: Applications of Perron-Frobenius theory to population dynamics. J. Math. Biol. 44, 450-462 (2002)

6. Li, X, Wang, W: A discrete epidemic model with stage structure. Chaos Solitons Fractals 26, 947-958 (2006)

7. De la Sen, M, Alonso-Quesada, S: Some equilibrium, stability, instability and oscillatory results for an extended discrete epidemic model with evolution memory. Adv. Differ. Equ. 2013, 234 (2013)

8. Caccetta, L, Rumchev, VG: A survey of reachability and controllability for positive linear systems. Ann. Oper. Res. 98, $101-122(2000)$

9. Berman, A, Plemons, RJ: Nonnegative Matrices in Mathematical Science. SIAM, Philadelphia (1994)

10. Diblík, J, Khusainov, D, Ruzicková, M: Controllability of linear discrete systems with constant coefficients and pure delay. SIAM J. Control Optim. 47, 1140-1149 (2008)

11. Diblík, J, Feckan, M, Pospísil, M: On the new control functions for linear discrete delay systems. SIAM J. Control Optim. 52, 1745-1760 (2014)

12. Bru, R, Romero, S, Sánchez, E: Canonical forms for positive discrete-time linear systems. Linear Algebra Appl. 310 , 49-71 (2000)

13. Farina, L, Rinaldi, S: Positive Linear Systems. Wiley, New York (2000)

14. Bru, R, Coll, C, Romero, S, Sánchez, E: Reachability indices of positive linear systems. Electron. J. Linear Algebra 11, 88-102 (2004)

15. Kajin, M, Almeida, PJAL, Vieira, MV, Cerqueira, R: The state of the art of population projection models: from the Leslie matrix to evolutionary demography. Oecol. Aust. 16(1), 13-22 (2012)

16. Leslie, PH: Some further notes on the use of matrices in population mathematics. Biometrika 35, $213-245$ (1948)

17. Muratori, S, Rinaldi, S: Equilibria, stability and reachability of Leslie systems with nonnegative inputs. IEEE Trans. Autom Control 35, 1065-1068 (1990)

18. Caswell, H: Matrix Population Models: Construction, Analysis and Interpretation. Sinauer, Sunderland (2001)

10.1186/1687-1847-2014-296

Cite this article as: Cantó et al.: On stability and reachability of perturbed positive systems. Advances in Difference

Equations 2014, 2014:296 